\title{
Drogadicção em Adolescentes: O papel interventivo da Família
}

\author{
Dorinaldo de Freitas Cintra Junior ${ }^{1^{*}}$; Brena Fernandes Custodio ${ }^{2}$; Hillary Negreiros Guimarães ${ }^{3}$ \\ Layon Peixoto Landim Correia ${ }^{4}$
}

\begin{abstract}
Resumo: O uso das drogas por adolescentes atualmente constitui-se numa ameaça à saúde pública. É durante esta etapa do desenvolvimento que a formação da personalidade e individualidade passa por expressivo delineamento, e é também o período em que as drogas se fazem mais presentes. O presente estudo, objetivou através de uma revisão de literatura apresentar a importância de conhecer as peculiaridades do uso de substâncias nessa fase de transição entre a puberdade e vida adulta e identificar fatores de risco e proteção associados à estrutura familiar dos adolescentes. Como resultados apresentamos estudos que entendem que seja fundamental o apoio e orientação familiar como possibilidades de prevenção e posvenção aos agravos inerentes a adolescência em exposição ao uso de Drogas. Conclui-se ainda, que é destacado o papel da família e o da educação como protetores de risco e principais bases na formação de características de resiliência e acesso as redes de cuidado.
\end{abstract}

Palavras-Chave: Família. Adolescência. Drogas. Fatores Protetores. Consumo

\section{Drug Addiction in Teens: The Family Active Role}

Abstract: The use of drugs by teenagers currently constitutes a threat to public health. It is during this stage of development that the formation of personality and individuality goes through expressive delineation, and is also the period in which drugs are more present. This study aimed through a literature review introduce the importance of knowing the peculiarities of substance use during this transition phase between puberty and adulthood and identify risk and protective factors associated with family structure of adolescents. As a result we present studies that believe the support and family counseling as possibilities for prevention and postvention inherent to adolescence diseases in exposure to the use of drugs is fundamental. We conclude also that is highlighted the role of the family and of education as risk protectors and major bases in the formation of resiliency features and access to care networks.

Keywords: Family. Adolescence. Drugs. Protective factors. Consumption.

\section{Introdução}

O uso das drogas atualmente constitui-se numa ameaça à saúde, sendo considerado pela Organização Mundial da Saúde (OMS) um problema mundial e, no Brasil, um problema de saúde pública que vem se alastrando, independentemente de idade, sexo e classe social.

\footnotetext{
${ }^{1}$ Psicólogo, Psicanalista, Mestrando em Psicologia pelo Programa de Pós-Graduação em Psicologia da Universidade de Fortaleza, Professor do Curso de Psicologia da Faculdade Vale do Salgado, dorinaldojr@gmail.com;

${ }^{2}$ Graduanda do Curso de Psicologia pela Faculdade Vale do Salgado - FVS, psicologia_bf@outlook.com;

${ }^{3}$ Graduando do Curso de Psicologia pela Faculdade Vale do Salgado - FVS, hillaryguimaraes_@hotmail.com;

${ }^{4}$ Graduando do Curso de Psicologia pela Faculdade Vale do Salgado - FVS, lyonpeixoto ico@ hotmail.com;

*Autor correspondente: Dorinaldo Cintra, rua A, nº88, Parque dois Irmãos, Fortaleza-CE, CEP: 60743-262.
} 
A palavra droga, segundo a Organização Mundial de Saúde (OMS), corresponde a toda a substância que introduzida no organismo vivo modifica uma ou mais das suas funções biológicas e, possivelmente, sua estrutura. Esta definição engloba substâncias ditas lícitas como bebidas alcoólicas, tabaco e certos medicamentos, e igualmente, as substâncias ilícitas como a cocaína, LDS, ecstasy, entre outras. Conforme Brasil (2011), milhões de pessoas morrem a cada ano em decorrência do consumo de produtos derivados do tabaco, cerca de 5 milhões somente pelo uso do tabaco, a estimativa da OMS utilizando os dados de mortalidade brasileiras de 2004, é de que em 30 anos, se mantida esse crescimento do consumo, no Brasil teremos 200 mil mortes por ano.

O hábito de fumar está associado à mortalidade por vários tipos de doenças, a exemplo do câncer (de pulmão, boca, laringe, faringe, estômago, colo do útero), da doença pulmonar obstrutiva crônica (DPOC), da doença coronariana, da hipertensão arterial e do acidente vascular cerebral (AVC) (BRASIL, 2011).

O uso de álcool por menores de idade está mais associado à morte do que todas as substâncias psicoativas ilícitas em conjunto. Sabe-se, por exemplo, que os acidentes automobilísticos são a principal causa de morte entre jovens dos 16 aos 20 anos (PECHANSKY, et al., 2004).

Segundo pesquisas apontadas por Pechansky (2004), estar alcoolizado aumenta a chance de violência sexual, tanto para o agressor quando para a vítima. A ligação entre sexo desprotegido e uso de álcool parece ser afetada pela quantidade de álcool consumida, interferindo na elaboração do juízo crítico. Da mesma forma, estando intoxicado, o adolescente envolve-se mais em atividades sexuais sem proteção, com maior exposição às doenças sexualmente transmissíveis, como ao vírus HIV, e maior exposição à gravidez.

Seibel e Junior (2001) também relatam que a maioria dos dependentes de drogas apresentaria um tipo de estruturação de personalidade depressiva, caracterizada por importante imaturidade afetiva e problemas de identidade, levando a um prolongamento da crise da adolescência, ficando o indivíduo a mercê das influências dos grupos de semelhantes.

Em vista disso, o conhecimento preciso de fatores associados ao uso de drogas em jovens no país é de grande relevância, pois permitiria intervenções sobre comportamentos e fatores de risco com vista a inibir o possível progresso de um uso pesado de drogas lícitas e ilícitas, vício progressivamente deletério para o jovem.

Assim o presente estudo, tem por objetivo discutir, à luz de alguns autores da literatura, o uso de substâncias psicoativas na adolescência, fase de transição entre a puberdade e vida adulta, bem como identificar fatores de risco e proteção associados à estrutura familiar dos adolescentes, cuja influência na formação dos indivíduos é inevitável.

Os grupos desenvolvem representações que, por sua vez, atuam sobre as representações sociais de muitos outros objetos, daí a importância da interação familiar no nosso estudo. 
Para entender a relação entre uso de drogas em adolescentes, torna-se de fundamental importância analisar algumas particularidades da adolescência. Esta fase de desenvolvimento compreende a transformação do jovem até a idade adulta, não apenas sob o ponto de vista biológico, como também cognitivo, emocional e social (MARTINS, 2011).

Caracterizar a adolescência somente como faixa etária, como mera divisão cronológica, seria, uma maneira muito simplista de observá-la, uma vez que ela compreende a transformação do jovem até a idade adulta. Compreendida como um período crítico de transição de vida é na adolescência que o sujeito vivência descobertas significativas, afirma sua personalidade, individualidade e segundo estudos apontados por Cavalcante e Barroso (2008) é também o período onde os mesmos estão mais propensos ao uso de drogas.

Em estudos, Freires e Gomes (2012) propõem um destaque a partir da ideia de que a vulnerabilidade e o risco podem ser melhor entendidos a partir do que se apresenta nos comportamentos dos adolescentes, principalmente a respeito da experimentação e uso precoce, em geral, por estarem relacionados a diversos fatores inerentes à juventude como a presença de: sensação de onipotência; busca de novas experiências; ser aceito pelo grupo, independência; desafio da estrutura familiar e social; conflitos psicossociais e existenciais; bem como aos aspectos relacionados à família, estrutura, apoio, presença de drogadição. Salienta-se ainda que existam outros aspectos que devem ser respeitados. Dentre eles, destacam-se: a facilidade de acesso, a permissividade e a falta de fiscalização no cumprimento das leis, além das questões próprias da cultura contemporânea ocidental.

Nesta fase, o conceito de interação grupal é perceptível e o adolescente busca pertencer a um grupo com o qual se identifica e poderá ter a capacidade de influenciar suas ações (CAVALCANTE; BARROSO, 2008). Os pares desempenham um papel importante na modelagem de comportamento. Estar associado a pares desviantes tem sido mostrado por consistência como um preditor ao uso abusivo do álcool (MARTINS, 2011).

A família também corrobora na constituição de vida do sujeito, visto que é no seio familiar onde se constroem valores e princípios que em confronto com o mundo e suas relações, dizem de todo o repertório de comportamento do indivíduo (GUIMARÃES, et. al., 2009). É no contexto familiar que o sujeito adquire de forma ativa os seus primeiros valores morais e seus padrões de comportamento. Segundo a afirmação de Freires e Gomes "Mesmo que o jovem passe a conviver mais em outros ambientes, a família ainda é um lugar privilegiado para a promoção da educação" (FREIRE; GOMES, 2012, p.102). Somente quando os valores morais, e fatores de proteção adquiridos pelo jovem em casa não são adequados, é que outros ambientes poderão exercer maior na vida do adolescente (GUIMARÃES, et al., 2008) 


\section{O Papel da Família e a Prevenção}

A partir da análise dos estudos apontados por Guimarães et al 2009, as famílias disfuncionais, ou seja, aquelas nas quais existe um funcionamento patológico com relação à comunicação, estabelecimento de regras e limites, e falta de afeto, costumam ser o tipo mais encontrado em adolescentes dependentes de drogas.

Em um estudo comparativo entre adolescentes dependentes e não dependentes de drogas, Pratta e Santos apud Guimarães (2009) revelam ser de fundamental importância à comunicação familiar, uma vez que esta funciona como um dos principais meios para uma relação satisfatória entre pais e filhos. Segundos esses autores "Um ambiente familiar no qual não existe espaço para expressar sentimentos, ideias e opiniões nem outra possibilidade de ser ouvido e/ou compreendido é apontado pelos adolescentes dependentes de drogas como um dos aspectos negativos da dinâmica familiar" (GUIMARÃES, 2009, p. 71).

Segundo ideias estabelecidas por Guimarães (2008), são crucias para a prevenção de comportamentos antissociais na adolescência, os fortes laços e papéis afetivos de pais com seus filhos. Estes rejeitariam que comportamentos como: roubo, uso de drogas e prostituição, por exemplo, se manifestassem. A ausência de monitoramento parental, ou seja, a falta de interesse que os pais demonstram em relação à vida de seus filhos, como os lugares que frequentam quem são seus amigos, se eles têm um bom desempenho na escola, bem como a ausência de uma hierarquia bem definida, corroboram como práticas influentes ao consumo e dependência de drogas por adolescentes.

Seria, portanto, a dinâmica familiar em que o jovem está inserido uma das causas conhecidas como facilitadoras para os comportamentos antissociais e desviantes (GUIMARÃES, 2008; LARANJEIRA 2004; MARTINS, 2011; RONZANI, 2009). Famílias mais distanciadas que não se envolvem em atividades conjuntas, que não acolhem seus filhos, podem levar o adolescente a se envolver com drogas e álcool e/ou com grupos de pares desviantes que fazem uso dessas substâncias, muitas vezes na tentativa de compensar o vazio deixado pela família.

Uma possível proposta de entendimento aponta que isso geralmente acontece, pois, pais e filhos encontram-se em momentos diferentes de transformação, ou seja, os adolescentes costumam questionar valores e regras familiares, preocupando-se intensamente com o futuro, enquanto seus pais se encontram em uma etapa de questionamento profissional, de reflexão e de transformação, também repensando o futuro. É possível pontuar, então, que existe um cruzamento entre o ciclo vital da família e o de seus membros, sendo que cada etapa envolve processos emocionais de transição, bem como mudanças primordiais para dar seguimento ao desenvolvimento tanto individual quanto familiar (PAIVA, 2009).

Segundo Guimarães (2008), outro fator de risco para tais condutas desviantes e certamente uma das grandes influências é o uso de drogas pelos pais e outros familiares. A falta de suporte 
parental, atitudes permissivas dos pais perante o consumo e incapacidade dos mesmos de controlar seus filhos, segundo estudos de Paiva e Ronzani (2009), são fatores predisponentes à iniciação ou continuação do uso de drogas pelos jovens.

O diálogo é a principal premissa que um ambiente familiar agradável e harmonioso pode gerar. A Família nessa perspectiva pode atuar no sentido de proteção à criança e/ou ao adolescente antes mesmo que ele tenha um primeiro contato com as drogas. Pois por meio do diálogo, os membros da família têm a possibilidade de aumentar sua proximidade. Tal aspecto transmite segurança tanto para os pais (pois estão atentos ao dia-a-dia dos filhos) quanto para os adolescentes (os quais se sentem seguros e valorizados pelos pais), pois a partir de uma relação mais próxima, é mais fácil para os pais detectarem mudanças no comportamento dos filhos (PRATTA, SANTOS, 2006).

Segundo estudos apontados por Freires e Gomes (2012), famílias que demonstram uma boa relação entre si, bem como aquelas que não consomem nenhum tipo de drogas lícitas ou ilícitas apresentam menor probabilidade de terem filhos adolescentes envolvidos com drogas ou que desenvolvam condutas antissociais: "Dessa forma, pode-se verificar que o bom funcionamento familiar, que tenha coesão e adaptabilidade moderadas, correlaciona-se positivamente com os fatores protetores e preventivos do consumo de drogas na adolescência”. (FREIRES; GOMES, 2012, p.102) Assim, a família está implicada no desenvolvimento saudável, ou não, de seus membros, já que ela é entendida como sendo o elo que os une às diversas esferas da sociedade.

\section{Considerações Finais}

Esse estudo procurou identificar o uso de drogas entre adolescentes e as práticas de monitoramento exercidas pelos pais, buscando entender tais práticas como fatores de proteção ou risco para o envolvimento do uso e abuso de drogas por adolescentes.

Acredita-se que este trabalho possa representar uma contribuição, na evolução dos conhecimentos sobre o papel desempenhado, direta ou indiretamente, pelos familiares sobre certos comportamentos considerados indesejáveis de seus filhos, muitas vezes atribuídos ao uso das drogas ou às chamadas más companhias.

Ao que se refere às ações relacionadas ao consumo de drogas por adolescentes, a família deve ser considerada. Além da família, os estudos consultados se preocuparam em compreender o impacto do grupo de pares desviantes no uso abusivo de drogas por adolescentes. Durante muito tempo, essa variável foi percebida como a principal influência no tocante à questão das drogas, porém atualmente, estudos evidenciam que a relação estabelecida com a família pode ser muito mais determinante. Os mesmos apontaram para a capacidade da família de minimizar o efeito de grupo de amigos e pares desviantes. 
Dados indicados pelas pesquisas no que se referem ao uso de drogas, apontam diferença significativa das práticas educativas positivas com relação às práticas negativas, sugerindo que as práticas positivas podem contribuir para o não envolvimento do adolescente com o uso de drogas. No entanto a aplicação das práticas educativas positivas é significativamente inferior à aplicação das práticas negativas exercidas pelos pais, visto que é crescente o aumento do consumo de drogas por adolescentes.

Com esse estudo não se pretende afirmar que a família é a única influencia para o envolvimento do adolescente com as drogas. Mas através dele torna-se legítima a importância de enfocar essa questão importante que é implicação das relações familiares, que não pode mais ser deixada de lado quando se aborda esse tema, seja no âmbito do tratamento ou mesmo da prevenção, atuando como fatores de risco e proteção.

\section{Referências}

BARROS, J. A. O campo da história: especialidades e abordagens. Petrópolis: Vozes, 2004.

BRASIL. Lei 8.069, de 13 de julho de 1990. Dispõe sobre o Estatuto da Criança e do Adolescente e dá outras providências. Disponível em: <http://www.planalto.gov.br/ ccivil_03/leis/L8069.htm>. Acesso em: 25. Maio 2015

CAVALCANTI, M.B.P.T; ALVES, M.D.S; BARROSO, M.G.T. Adolescência, álcool e drogas: uma revisão na perspectiva da promoção da saúde. Esc. Anna Nery Ver. Enferm. 12(3), 2008, pp 555-59

CECCONELLO, A. M; ANTONI, C.; KOLLER, S. H. Práticas Educativas, Estilos Parentais e Abuso Físico no Contexto Familiar. Psicologia em Estudo, Maringá, v.8, num. esp., p. 45-54, 2003. Disponível em http://www.scielo.br/pdf/pe/v8nspe/v8nesa07, acessado em: 01/06/2015.

FERREIRA, V.M; FILHO, E.A.S. Maconha e contexto familiar: um estudo psicossocial entre universitários do Rio de Janeiro. Psicologia \& Sociedade; 19 (1): 52-60; jan/abr, 2007.

FERREIRA, V.R.T; COLOGNESE, B. T. Prejuízos de funções executivas em usuários de cocaína e crack. Avaliação Psicológica, 2014, 13(2), pp. 195-201.

FREIRES, Irlan de Almeida; GOMES, Edézia Maria de Almeida. O papel da família na prevenção ao uso de substâncias psicoativas. Rev. bras. ciênc. saúde;16(1), mar. 2012.

FILHO, A.J.A; FEREEIRA, M.A; GOMES, M.L.B; SILVA, R.C; SANTOS,T.C.F. O adolescente e as drogas: Consequências para a saúde. Esc Anna Nery. Rev Enferm. dez; 11 (4): 605 - 10, 2007.

GUIMARÃES, A.B.P; HOCHGRAF, P.B; BRASILIANO, S; INGBERMAN, Y.K. Aspectos familiares de meninas adolescentes dependentes de álcool e drogas. Rev Psiq Clín. 2009;36(2):69-74, 2008.

HORTA, R. L.; HORTA, L.B.; PINHEIRO, R.T. Drogas: famílias que protegem e expõem adolescentes ao risco. J. Bras. Psiquiatr., 55(4):268-272, 2006. 
LARANJEIRA, R. Prefácio. In I. Pinsky \& M. A. Bessa. Adolescência e drogas (pp. 9-10). São Paulo: Contexto. 2004.

LIMA, T. C. S.; MIOTO, R. C.T. Procedimentos metodológicos na construção do conhecimento científico: a pesquisa bibliográfica. Rev. katálysis, Florianópolis, v. 10, n. spe, 2007.

MARQUES, A.C.P.R; CRUZ, M.S. O adolescente e o uso de drogas. Rev Bras Psiquiatr ;22 (Supl II):32-6. 2000.

MARTINS, M.A - Práticas educativas parentais e o uso de drogas entre os adolescentes escolares. Ribeirão Preto, 2011.

OMS, ORGANIZAÇÃO MUNDIAL DE SAÚDE. Classificação Estatística Internacional de Doenças e Problemas Relacionados à Saúde / CID-10. Ed 10. Geneva: Centro Colaborador da OMS para a Classificação de Doenças em Português - CBCD, 1993.

PAIVA, F.S; RONZANI, T.M. Estilos parentais e consumo de drogas entre adolescentes: Revisão sistemática. Psicologia em Estudo, Maringá, v. 14, n. 1, p. 177-183, jan./mar, 2009.

PINHO, P.H; OLIVEIRA, M.A; ALMEIDA, M.M. A reabilitação psicossocial na atenção aos transtornos associados ao consumo de álcool e outras drogas: uma estratégia possível?. Rev. Psiq. Clín, 35, supl 1; 82-88, 2008.

PRATA, E.M.M; SANTOS, M.A. Opiniões dos adolescentes do ensino médio sobre o relacionamento familiar e seus planos para o futuro. Paidéia, 2007, 17(36), 103-114.

PRATA, E.M.M; SANTOS, M.A. Reflexões sobre as relações entre drogatição, adolescência e família: um estudo bibliográfico. Estudos de Psicologia, 2006, 11(3), 315-322

PRATTA, E. M. M.; SANTOS, M. A. FAMÍLIA E ADOLESCÊNCIA: A Influência do Contexto Familiar no Desenvolvimento Psicológico de Seus Membros. Psicologia em Estudo, Maringá, v. 12, n. 2, p. 247-256, maio/ago. 2007. Disponível em http://www.scielo.br/pdf/pe/v12n2/v12n2a0 5 acessado em: 01/06/2015.

PECHANSKY, F; SZOBOT, M. C.; SCIVOLETTO, S. Uso de álcool entre adolescentes: conceitos, características epidemiológicas e fatores etiopatogênicos. Rev Bras Psiquiatr 2004;26(Supl I):14-17. Disponível em: http://www.scielo.br/pdf/rbp/v26s1/a05v26s1. Acessado em: 01/06/2015

SEIBEL S.D; JÚNIOR A.T. Dependência de drogas. São Paulo (SP): Atheneu; 2001 [online]. Disponível

em: http://www.mpsp.mp.br/portal/page/portal/infânciaahome_c/dr_drogadicao/dr_doutrina_drogadicao/G uia\%20Pratico\%20sobre\%20\%20Uso\%20e\%20Dependencia\%20de\%20Drogas.pdf. Acessado em: $02 / 06 / 2015$.

Fontes de Apoio: Coordenação de Pesquisa e Extensão da Faculdade Vale do Salgado - FVS.

Agradecimentos: Aos membros da Extensão Adolescência e Cidadania: Estudos relacionados a tecnologias e intervenções de valorização da vida - ACERTIVA. 


\section{Como citar este artigo (Formato ABNT):}

CINTRA JÚNIOR, D.F.; CUSTÓDIO, B.F.; GUIMARÃES, H.N.; CORREIA, L.P.L. Drogadicção em Adolescentes: O papel interventivo da família. Id on Line Revista de Psicologia, Novembro de 2015, vol.9, n.28. p. 111-118. ISSN 1981-1189.

Recebido: 14/09/2015

Aceito:17/09/2015 